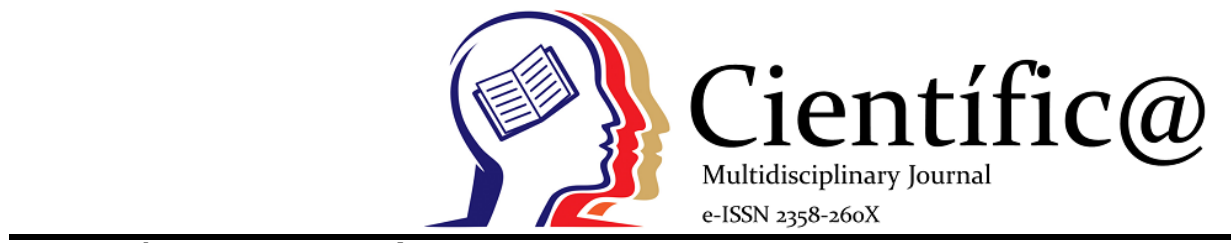

\title{
DINÂMICA DO FÓSFORO EM SOLOS DE ALTA FERTILIDADE: FONTES E DOSES FOSFATADAS EM CULTIVO DA CULTURA DE SOJA NO CERRADO
}

\section{PHOSPHORUS DYNAMICS IN HIGH FERTILITY SOILS: PHOSPHATE SOURCES AND DOSES IN CULTIVATION OF SOY CULTURE IN THE CERRADO}

\author{
José Mateus Kondo Santini*1; Salatiér Buzetti2; Adriano Perin3; Carlos Frederico de Souza \\ Castro4; Leonnardo Cruvinel Furquim $^{5}$; Daniel Noe Coaguila Nunez ${ }^{6}$; Flávio de Oliveira \\ Silveira7; Luiz César Lopes Filho8; Anderson Cruvinel Cabral ${ }^{9}$
}

\footnotetext{
${ }^{1}$ Autor para correspondência; Engenheiro Agrônomo, Dr., Professor do Instituto de Ensino Superior de Rio Verde santini@faculdadeobjetivo.com.br*;

${ }^{2}$ Engenheiro Agrônomo, Dr., Professor da Universidade Estadual Paulista - Faculdade de Engenharia de Ilha Solteirasbuzetti@agr.feis.unesp.br;

${ }^{3}$ Engenheiro Agrônomo, Dr., Professor do Instituto Federal Goiano - Câmpus Rio Verde-perinrj@yahoo.com.br;

${ }^{4}$ Químico, Dr., Professor do Instituto Federal Goiano - Câmpus Rio Verde-carlosfscastro@gmail.com;

${ }^{5}$ Engenheiro Agrônomo, Me. Professor do Instituto de Ensino Superior de Rio Verde-

leonnardo.cruvinel@faculdadeobjetivo.com.br;

${ }^{6}$ Biólogo, Dr. Professor do Instituto de Ensino Superior de Rio Verde-tuheraldo@gmail.com;

${ }^{7}$ Engenheiro Agrônomo, Consultor técnico da empresa Exacta-exacta.ai@gmail.com;

${ }^{8}$ Engenheiro Agrônomo Me. Professor do Instituto de Ensino Superior de Rio Verde-lopesfilholuizcesar@gmail.com;

${ }^{9}$ Acadêmico do curso de Engenharia agronômica do Instituto de Ensino Superior de Rio Verdeandersoncruvinelrv@gmail.com.
}

Info

Recebido: 21/06/2019

Publicado: $10 / 02 / 2020$

DOI: $10.29247 / 2358-260 X .2019 v 6 i 2 . p 14-23$

ISSN: 2358-260X

Palavras-Chave
Adubos, fertilidade do solo, Glycine max (L.)
Merrill.
Keywords:
Fertilizers, soil fertility, Glycine $\max$ (L.)

\section{Resumo}

A cultura da soja é uma das principais culturas do agronegócio brasileiro, podendo ser limitada por adubações equivocadas em área de cerrado. Com o presente trabalho, objetivou avaliar a dinâmica da adubação fosfatada, pelo uso de fontes e doses na adubação de manutenção da cultura da soja, em solo com alto teor de $\mathrm{P}$, verificando a real eficácia do uso do superfosfato triplo de eficiência aumentada. 0 experimento foi realizado em delineamento em blocos ao acaso, com quatro repetições, em esquema fatorial de $2 \times 5$, sendo o primeiro fator fontes de fertilizantes (superfosfato triplo e superfosfato triplo de eficiência aumentada) e segundo fator doses de $\mathrm{P}_{2} \mathrm{O}_{5}$ $\left(0,40,80,120\right.$ e $\left.240 \mathrm{~kg} \mathrm{ha}^{-1}\right)$. 0 experimento foi conduzido em área experimental, em Rio Verde, GO, buscando-se avaliar as influências nas características de componentes de produção e produtividade da soja, bem como, na dinâmica do P no solo. Constatou-se que a cultura da soja não respondeu à adubação fosfatada, em solo com alto teor de P. 0 uso do Superfosfato triplo de eficiência aumentada não modifica a produtividade de grãos, mas, aumentou a exportação de P via grãos da cultura, em comparação ao Superfosfato Triplo convencional. Para a adubação fosfatada, recomenda-se o uso do Superfosfato triplo convencional na dose de $75 \mathrm{~kg} \mathrm{ha}^{-1} \mathrm{de}_{2} \mathrm{O}_{5}$.

\footnotetext{
Abstract

The soybean crop is of the main crops of Brazilian agribusiness, and may be limited by wrong fertilization in cerrado. With this study aimed to evaluate the dynamics of phosphate fertilizer, the use of fertilization sources and doses in soybean maintenance, on soil with high content of $\mathrm{P}$, and checking the actual effectiveness of the use of triple superphosphate increased efficiency. The experiment was conducted in completely randomized block design with four replications in a factorial scheme $2 \times 5$, being the first factor sources of fertilizers (triple superphosphate and triple increased efficiency superphosphate) and second factor five doses of $\mathrm{P}_{2} \mathrm{O}_{5}(0,40,80$, 120 and $240 \mathrm{~kg} \mathrm{ha}^{-1}$ ). The experiment was conducted at the experimental area in Rio Verde, GO, seeking to evaluate the influences on the characteristics of production components and soybean yields, soil fertility, nutrient concentrations of harvested soybeans and the dynamics of $\mathrm{P}$ in the soil. For phosphate fertilizer, it is recommended the use of conventional triple superphosphate at dose of $75 \mathrm{~kg} \mathrm{ha}^{-1}$ of $\mathrm{P}_{2} \mathrm{O}_{5}$.
} 


\section{INTRODUÇÃO}

A cultura da soja (Glycine max (L.) Merrill) com sua elevada participação no agronegócio brasileiro, ocupa lugar de destaque nas exportações dos produtos agrícolas e na área destinada à agricultura.

Em busca de melhorias em produtividade de grãos, a correta nutrição das plantas, via fertilizantes, é de grande valia. Entre os nutrientes, o fósforo (P), apesar de ser requerido em pequenas quantidades pelas plantas, é um dos nutrientes mais limitantes à produção agrícola brasileira, devido seu baixo teor disponível e sua facilidade de fixação pelos coloides do solo (Araújo, 2011).

Uma tecnologia que vem sendo estudada como mitigadora da fixação de $\mathrm{P}$, é pelo uso de fertilizantes de eficiência aumentada, como os fertilizantes com revestimento de polímeros, que possuem liberação gradual do nutriente, podendo contribuir na sincronia de liberação do nutriente (Blaylock, 2007) de acordo com as necessidades nutricionais das plantas ao longo do ciclo de cultivo, possibilitando a redução dos efeitos de fixação do P ao solo (Valderrama et al, 2009) e, por consequência, maiores produtividades de grãos ou redução da necessidade de aplicação dos fertilizantes fosfatados, fato este, de grande interesse, devido a matéria prima de fertilizantes fosfatados serem finitas.

$\mathrm{Na}$ correta recomendação da adubação fosfatada, não somente a fonte é de interesse, mas, também, a dose de aplicação, perfazendo com que doses abaixo da necessária ocasione a degradação química do solo e doses superiores ocasione ao desprendimento monetário não necessário dos agricultores. Para a correta recomendação e adubação de cada cultura, se faz necessário avaliar não somente os reflexos diretos dos fertilizantes nos componentes de produção e produtividade, mas, avaliar a dinâmica do nutriente no sistema de cultivo.
Destaca-se também, que no melhoramento vegetal, visando o aumento de produtividade de grãos da soja, pode ter levado a seleção indireta de cultivares com alta responsividade ao uso de fertilizantes, já que nos campos de melhoramento, na seleção de novas cultivares, isolam-se todos os fatores de estresse, inclusive, os fatores nutricionais (Caixeta, 2012), utilizando-se elevadas doses de aplicação de fertilizantes, podendo ocasionar a seleção de cultivares modernas de baixa eficiência nutricional (Duncan e Baligar, 1990).

Assim sendo, é compreensível que o uso de doses de fertilizantes fosfatados, mesmo em solo com alta fertilidade, pode ocasionar efeitos positivos na produtividade de soja, bem como, o uso de fertilizante fosfatados de maior eficiência podem contribuir para melhoria da produtividade ou na redução do uso de adubos, mantendo o mesmo teor de $\mathrm{P}$ inicial, com dose de aplicação inferior ao fertilizante convencional.

Com o presente trabalho objetivou-se avaliar a dinâmica da adubação fosfatada pelo uso de fontes e doses, na adubação de manutenção da cultura da soja, em solo com teor alto de $\mathrm{P}$, verificando a real eficácia do uso do superfosfato triplo de eficiência aumentada.

\section{MATERIAL E MÉTODOS}

$\mathrm{O}$ experimento foi conduzido à campo no município de Rio Verde, GO, com localização geográfica entre os paralelos $20^{\circ} 45^{\prime}$ de latitude sul e $51^{\circ}$ 55 ' de longitude oeste, com altitude de $748 \mathrm{~m}$. A caracterização físico-química foi à seguinte: $\mathrm{pH}$ (em água $)=5,6 ; \mathrm{MO}=35,0 \mathrm{~g} \mathrm{dm}^{-3} ; \mathrm{P}($ Mehlich $\mathrm{I})=41,0$ $\mathrm{mg} \mathrm{dm}{ }^{-3} ; \mathrm{K}=6,7 \mathrm{mmol}_{\mathrm{c}} \mathrm{dm}^{-3} ; \mathrm{Ca}=37,8 \mathrm{mmol}_{\mathrm{c}} \mathrm{dm}^{-3}$; $\mathrm{Mg}=17,3 \mathrm{mmol}_{\mathrm{c}} \mathrm{dm}^{-3} ; \mathrm{S}=3,1 \mathrm{mg} \mathrm{dm}^{-3} ; \mathrm{Al}=0,0$ mmol $_{\mathrm{c}} \mathrm{dm}^{-3} ; \mathrm{V}=53,9 \%$ e textura média $\left(333 \mathrm{~g} \mathrm{~kg}^{-1} \mathrm{de}\right.$ argila, $166 \mathrm{~g} \mathrm{~kg}^{-1}$ de silte e $501 \mathrm{~g} \mathrm{~kg}^{-1}$ de areia).

A precipitação pluvial acumulada mensal durante o experimento foi de: outubro (2011): $232 \mathrm{~mm}$; 
novembro (2011): $76 \mathrm{~mm}$; dezembro (2011): $165 \mathrm{~mm}$; janeiro (2012): $275 \mathrm{~mm}$; fevereiro (2012): $265 \mathrm{~mm}$; março (2012): $125 \mathrm{~mm}$.

O experimento foi conduzido em delineamento em blocos ao acaso, com quatro repetições, em esquema fatorial de $2 \times 5$. O primeiro fator foi constituído de fontes de fertilizantes (superfosfato triplo e superfosfato triplo de eficiência aumentada) e o segundo fator doses de aplicação $(0,40,80,120$ e 240 $\mathrm{kg} \mathrm{P}_{2} \mathrm{O}_{5} \mathrm{ha}^{-1}$ ) de fertilizantes fosfatados, no sulco de semeadura.

Antecedendo a semeadura do experimento realizou-se a dessecação da área, em 21/10/2011, utilizando herbicida glyphosate, na dose de $960 \mathrm{~g}$ i.a ha 1. As sementes do cultivar de soja NA 7337 RR foram tratadas com Imidacloprid e Thiamethoxam, ambos na dosagem de 105 g i.a para cada $100 \mathrm{~kg}$ de sementes de soja. Posteriormente, foi realizada a inoculação da soja com Bradyrhizobium japonicum na dosagem de 200 gramas de inoculante (turfa moída) para $40 \mathrm{~kg}$ de sementes, sendo considerada a concentração mínima de $10^{8}$ células viáveis por grama de turfa. Simultaneamente à semeadura foram aplicados $100 \mathrm{~kg} \mathrm{~K}_{2} \mathrm{O} \mathrm{ha} \mathrm{ha}^{-1}$ de, na forma de $\mathrm{KCl}$, e $\mathrm{P}_{2} \mathrm{O}_{5}$, de acordo com cada tratamento.

Cada unidade experimental foi composta por cinco linhas, espaçadas entre si $0,45 \mathrm{~m}$, com cinco metros de comprimento. Considerou-se como área útil da parcela as três fileiras centrais e desprezou-se $0,5 \mathrm{~m}$ das extremidades, totalizanto $5,4 \mathrm{~m}^{2}$. A semeadura foi realizada em 29/10/2011, distribuindo-se 18 sementes por metro no sulco de semeadura, utilizando a semeadora-adubadora.

O controle de plantas daninhas em pós-emergência foi realizado com o uso do herbicida glyphosate, na dose de 960 g i.a ha-1 aos 15 e 40 dias após a emergência (DAE). Também foi realizado o controle da lagarta da soja (Anticarsia gemmatalis) e lagarta falsa-medideira (Pseudoplusia includens) aos 45 DAE com Teflubenzurom
(50 mL ha-1). A partir dos 60 DAE, procurou-se identificar focos de ferrugem asiática, neste período, foi aplicado o fungicida trifoxystrobin + ciproconazole $\left(300 \mathrm{~mL} \mathrm{ha}^{-1}\right)$, preventivamente, uma vez que a doença não foi encontrada. Doze dias após a primeira aplicação preventiva para controle de ferrugem asiática, outra amostragem foi realizada no experimento e constatado o foco da doença, efetuou-se então, o controle com o fungicida azoxystrobin + ciproconazole $\left(300 \mathrm{~mL} \mathrm{ha}{ }^{-1}\right)$, realizando essa mesma prática aos $85 \mathrm{DAE}$.

Foram realizadas as avaliações de produtividade de massa fresca (MF) e seca (MS) de parte aérea das plantas da soja, procedendo a coleta aos 42 DAE (estádio fenológico $\mathrm{V}_{2}$ ), realizando-se o corte rente ao solo, contidas em 1,3 m de fileira. Após a coleta, as plantas foram pesadas em balança de precisão, para determinação da massa fresca total, e convertidos os dados para $\mathrm{kg} \mathrm{ha} \mathrm{h}^{-1}$. Para quantificar a produção de massa seca da parte aérea das plantas, o material coletado foi acondicionado em estufa de ventilação forçada à de $65^{\circ} \mathrm{C}$ por 72 horas, procedendo, em posterior, a pesagem e conversão dos dados para kg ha1 .

Para a avalição de número de vagens por plantas (NVP), procedeu-se a quantificação das vagens de dez plantas aleatórias por parcela, no ato da colheita, sendo estimado o NVP pela média aritmética das plantas amostradas.

$\mathrm{Na}$ análise de massa de 1000 grãos (M1000) e de produtividade de grãos (Prod), o material foi coletado manualmente e, com o auxílio de uma trilhadora mecânica, o material foi trilhado. O material coletado foi pesado, para estimar a produtividade de grãos, sendo em seguida convertidos para $\mathrm{kg} \mathrm{ha-1}$, com seu valor corrigido para 13\% de umidade. Para a análise de N1000, os grãos foram limpos para remoção de impurezas, homogeneizados e posteriormente amostrados quatro lotes de 1000 grãos, aleatoriamente 
por parcela, e com o auxílio de uma balança de precisão os grãos foram pesados, averiguando-se o peso dos mesmos.

Ao término da colheita da soja, realizou-se a amostragem de solo, de forma aleatória, na camada de 0-0,2 m de profundidade, retirando três amostras simples de cada parcela, para formação de uma amostra composta. Com as amostras retiradas, procedeu-se a análise de P no solo de acordo com Raij et al. (2001).

$\mathrm{Na}$ análise de exportação de fósforo, com as amostras devidamente secas, em estufa, e moídas, com moinho do tipo Wiley, foram analisadas as concentrações nutricionais do elemento e posterior convertidos em $\mathrm{kg} \mathrm{ha}^{-1}$, baseando-se na produtividade de cada parcela. Na análise de $\mathrm{P}$ no tecido dos grãos, utilizou-se a metodologia descrita por Malavolta et al. (1997).

Para a comparação de médias dos tratamentos, inicialmente, realizou-se o teste de Kolmogorov Smirnov, buscando avaliar a homocedasticidade (5\%) das variáveis, nos casos em que o $\mathrm{H}_{0}$ foi rejeitado, efetuou a transformação de dados utilizando a equação $(x+0,5)^{0,5}$. Posteriormente, os dados foram submetidos à análise de variância utilizando o pacote ExpDes do software R 3.2.2 programa estatístico R (Ferreira et al., 2011). Quando verificado efeito significativo para as variáveis qualitativas, foi realizada a comparação de médias pelo teste de Tukey ( $\mathrm{p}<0,05)$, já para as variáveis quantitativas foram realizadas regressões polinomiais (de primeiro e segundo grau) à 5\% e 1\% de probabilidade.

\section{RESULTADOS E DISCUSSÃO}

Nas variáveis de componentes agronômicos e produtividade de grãos (MF, MS, NVP, M1000 e Prod) não foram observados efeitos significativos para as fontes e doses avaliadas, bem como, não houve interação significativa entre as fontes e doses testadas (Tabela 1).

Tabela 1. Média, desvio padrão amostral (s), coeficiente de variação (CV) e teste F da produtividade de matéria fresca (MF) e seca (MS), número de vagens por planta (NVP), peso de 1000 grãos (p1000) e produtividade de grãos de soja (Prod.), submetido a fontes (Superfosfato triplo convencional; Superfosfato triplo revestido por polímeros) e doses $\left(0 ; 40 ; 80 ; 120\right.$; e $\left.240 \mathrm{~kg} \mathrm{P}_{2} \mathrm{O}_{5} \mathrm{ha}^{-1}\right)$ de aplicação de fertilizantes fosfatados, em sulco de semeadura da cultura da soja.

\begin{tabular}{|c|c|c|c|c|c|c|}
\hline \multicolumn{2}{|l|}{ Variáveis } & $\begin{array}{l}\text { MF } \\
-(k\end{array}$ & MS & NVP & $\begin{array}{l}\text { M1000 } \\
\text { (g) }\end{array}$ & $\begin{array}{l}\text { Prod } \\
\left(\mathrm{kg} \mathrm{ha}^{-1}\right)\end{array}$ \\
\hline \multicolumn{2}{|l|}{ Média } & 11794,8 & 2538,8 & 57,9 & 146,1 & 3875,2 \\
\hline \multicolumn{2}{|l|}{$\mathrm{s}$} & 1927,6 & 452,1 & 8,0 & 5,8 & 436,1 \\
\hline \multicolumn{2}{|l|}{ CV (\%) } & 16,3 & 17,8 & 13,8 & 4,0 & 11,3 \\
\hline \multicolumn{7}{|l|}{ Teste F } \\
\hline \multicolumn{2}{|l|}{ Fontes $(\mathrm{F})$} & $1,172^{\text {ns }}$ & $0,483^{\text {ns }}$ & $0,137^{\mathrm{ns}}$ & $0,285^{\text {ns }}$ & $0,870^{\text {ns }}$ \\
\hline \multirow{3}{*}{ Doses (D) } & $\mathrm{x}$ & $1,295^{\mathrm{ns}}$ & $0,443^{\text {ns }}$ & $1,642^{\text {ns }}$ & $0,106^{\text {ns }}$ & $1,160^{\mathrm{ns}}$ \\
\hline & $x^{2}$ & $2,474^{\text {ns }}$ & $1,331^{\mathrm{ns}}$ & $1,088^{\text {ns }}$ & $0,076^{\mathrm{ns}}$ & $0,726^{\mathrm{ns}}$ \\
\hline & & $0,104^{\mathrm{ns}}$ & $0,206^{\mathrm{ns}}$ & $0,523^{\text {ns }}$ & $1,557^{\mathrm{ns}}$ & $2,220^{\text {ns }}$ \\
\hline
\end{tabular}

ns: Não significativo ( $p \geq 0,05)$.

Não foi observada interação entre as fontes e as doses estudadas $\left(0 ; 40 ; 80 ; 120\right.$; e $\left.240 \mathrm{~kg} \mathrm{P}_{2} \mathrm{O}_{5} \mathrm{ha}^{-1}\right)$ (Tabela 2) nas variáveis avaliadas de dinâmica de P (P residual no solo [0-0,2m; P-res]; exportação total de P via grãos [P-exp]; e P fixado [0-0,2m; P-fix]). Para as fontes avaliadas não se verificou efeito significativo no P-res e P-fix, mas, houve efeito significativo para o Pexp. Já para as doses testadas, todas as variáveis da dinâmica de $\mathrm{P}$ analisadas, ajustaram nas doses em estudo (Tabela 2). 
Tabela 2. Concentração Média, desvio padrão amostral (s), coeficiente de variação (CV) e teste $\mathrm{F}$ da dinâmica de P (P residual no solo [0-0,2m; P-res], exportação total de P via grãos [P-exp] e P fixado [0-0,2m; P-fix]), sob aplicação de fontes (Superfosfato triplo convencional; Superfosfato triplo revestido por polímeros) e doses (0; 40; 80; 120; e $240 \mathrm{~kg} \mathrm{P}_{2} \mathrm{O}_{5} \mathrm{ha}^{-1}$ ) de fertilizantes fosfatados, em sulco de semeadura da cultura da soja.

\begin{tabular}{|c|c|c|c|c|}
\hline \multicolumn{2}{|l|}{ Variáveis } & $\begin{array}{l}\text { P-res } \\
(\mathrm{kg} \mathrm{P} \mathrm{ha-1})\end{array}$ & P-exp & P-fix \\
\hline \multicolumn{2}{|l|}{ Média } & 94,4 & 19,1 & $5,3^{\#}$ \\
\hline \multicolumn{2}{|l|}{ s } & 29,5 & 0,9 & 2,5 \\
\hline \multicolumn{2}{|l|}{ CV $(\%)$} & 31,2 & 4,6 & 47 \\
\hline \multicolumn{5}{|l|}{ Teste F } \\
\hline \multicolumn{2}{|l|}{ Fontes $(\mathrm{F})$} & $1,030^{\text {ns }}$ & $5,366^{*}$ & $4,034^{\mathrm{ns}}$ \\
\hline \multirow{3}{*}{$\begin{array}{l}\text { Doses } \\
\text { (D) }\end{array}$} & $\mathrm{x}$ & $12,181^{* *}$ & $2,544^{\mathrm{ns}}$ & $34,179^{* *}$ \\
\hline & $x^{2}$ & $5,974^{* *}$ & $4,576^{*}$ & $16,774^{* *}$ \\
\hline & $x^{3}$ & $4,861^{* *}$ & $3,902^{*}$ & $12,404^{* *}$ \\
\hline $\mathrm{F} \times \mathrm{D}$ & & $0,517^{\mathrm{ns}}$ & $2,151^{\mathrm{ns}}$ & $0,682^{\mathrm{ns}}$ \\
\hline
\end{tabular}

ns: Não significativo; ${ }^{* * *}$ : Significativo a $5 \%$ e 1\%, respectivamente; \#: Dados corrigidos seguindo a equação $\left(x^{2}+0,5\right)^{0,25}$.

As médias dos tratamentos para as variáveis de eficiência aumentada (SFTEA) foi superior ao dinâmica de P são citadas na Tabela 3. Como ressaltado, superfosfato triplo convencional (SFTC), com $0,5 \mathrm{~kg}$ de verificou-se efeito significativo para o P-exp, em relação $\quad \mathrm{P} \mathrm{ha}^{-1}$ a mais exportado.

as fontes testadas, sendo que o superfosfato triplo de

Tabela 3. P residual no solo (0-0,2m; P-res), exportação total de P via grãos (P-exp) e P fixado (0-0,2m; P-fix), submetidos às fontes (superfosfato triplo convencional (SFTC); superfosfato triplo de eficiência aumentada (SFTEA)) de fertilizantes fosfatados, na cultura da soja, em sulco de semeadura.

\begin{tabular}{llll}
\hline Fontes & $\begin{array}{l}\text { P-res } \\
\left(\mathrm{kg} \mathrm{P} \mathrm{ha}{ }^{-1}\right)\end{array}$ & P-exp & P-fix $^{\#}$ \\
\hline SFTEA & $90,1^{\text {ns }}$ & $18,8^{*}$ & $35,5^{\text {ns }}$ \\
SFTC & 98,7 & 19,3 & 27,4 \\
\hline
\end{tabular}

ns: Não significativo; *: Significativo a 5\%; \#: Dados corrigidos seguindo a equação $(x+0,5)^{0,5}$.

Nas doses avaliadas, as três variáveis da dinâmica solo, sendo que, a partir dessa dose, inicia-se o do P (P residual; P exportado e P fixado; Figura 1) acréscimo de $0,422 \mathrm{~kg} \mathrm{ha}^{-1}$ de $\mathrm{P}$ para cada $\mathrm{kg}$ de $\mathrm{P}_{2} \mathrm{O}_{5}$ ajustaram de modo cúbico $\left(x^{3}\right)$ (Figura 1). Em avaliação aplicado, com teor máximo na dosagem de 193,3 kg hado ajuste do P-res, nota-se que até a dose de $74,7 \mathrm{~kg}$ ha- $\quad{ }^{1}$ de $\mathrm{P}_{2} \mathrm{O}_{5}$.

1 de $\mathrm{P}_{2} \mathrm{O}_{5}$ não houve incremento nos teores de $\mathrm{P}$ do 


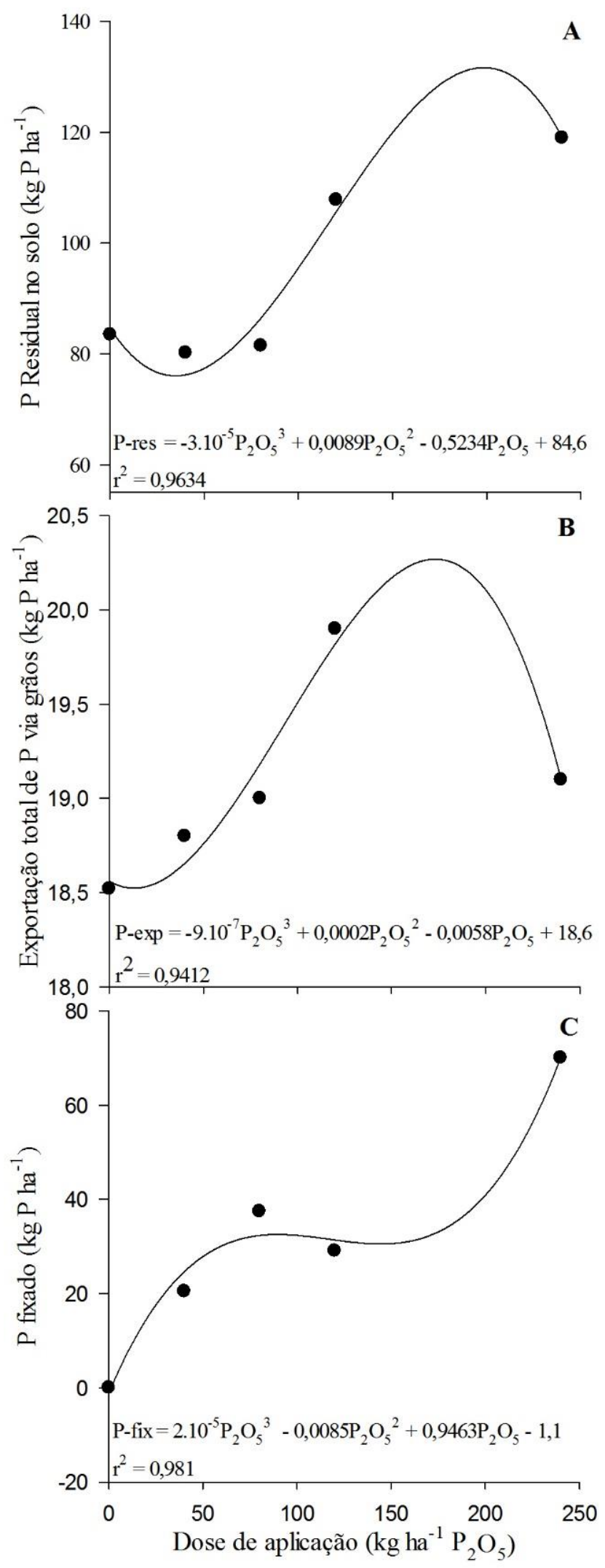

Figura 1. Dinâmica de P (P residual no solo [A]; exportação total de P via grãos [B]; e P fixado [C]) sob aplicação de doses $\left(0 ; 40 ; 80 ; 120\right.$; e $240 \mathrm{~kg} \mathrm{P}_{2} \mathrm{O}_{5}$ ha $\left.^{-1}\right)$ de fertilizantes fosfatados, em sulco de semeadura, na cultura da soja em cerrado. 
O incremento de P na variável P-exp se inicia posterior a dose de $33 \mathrm{~kg} \mathrm{ha}^{-1}$ de $\mathrm{P}_{2} \mathrm{O}_{5}$, com aumento de $12,4 \mathrm{~g} \mathrm{ha}^{-1}$ de $\mathrm{P}$ por $\mathrm{kg}$ de $\mathrm{P}_{2} \mathrm{O}_{5}$ aplicado, com máxima exportação (21,0 $\mathrm{kg} \mathrm{ha}^{-1}$ de $\left.\mathrm{P}\right)$, estimada, na dose de 187,5 $\mathrm{kg} \mathrm{ha}^{-1}$ de $\mathrm{P}_{2} \mathrm{O}_{5}$. Já para o P-fix, nota-se que, inicialmente, houve fixação de $\mathrm{P}(0,684 \mathrm{~kg}$ de $\mathrm{P}$ fixado por $\mathrm{kg}_{2} \mathrm{P}_{2} \mathrm{O}_{5}$ aplicado) até a dose de $75,7 \mathrm{~kg} \mathrm{ha}^{-1}$ de $\mathrm{P}_{2} \mathrm{O}_{5}$, posterior a esta dose, a fixação estabilizou até a dose de 155,3 kg ha-1 de $\mathrm{P}_{2} \mathrm{O}_{5}$, onde retornou à fixação de $\mathrm{P}$, fixando $0,439 \mathrm{~kg}$ de $\mathrm{P}$ por $\mathrm{kg} \mathrm{P}_{2} \mathrm{O}_{5}$ aplicado.

A ausência de efeitos significativos para as variáveis de componentes agronômicos (MF, MS, NVP e p1000), bem como para a produtividade de grãos (Tabela 1), são explicados devido ao teor de $\mathrm{P}$ considerado alto, de acordo com Souza e Lobato (2004), para a cultura da soja. Assim, para a cultivar de soja avaliada (NA 7337 RR), o aumento acentuado de P no solo, pouco influenciou em seus componentes de produção, tal forma, para a produtividade de grãos, perfazendo assim, com que a adubação de $\mathrm{P}$ com doses elevadas resulte apenas no desprendimento monetário do agricultor, sem retorno de capital.

Inerente ao resultado, pode-se afirmar que no melhoramento vegetal (do cultivar em estudo), visando o aumento de produtividade de grãos da soja, não resultou na seleção indireta de cultivares altamente responsivas a adubação fosfatada, ou seja, a cultivar avaliada não responde de modo acentuado à crescente adubação (Duncan \& Baligar, 1990).

Resultados na literatura de incrementos de produtividade na cultura da soja, com o uso da adubação fosfatada, condizem com o encontrado no presente trabalho, sendo que, em solos com teores baixos ou médios de $\mathrm{P}$ há resposta à adubação fosfatada (Valadão Junior et al., 2008; Alcântara Neto et al., 2010) e em solos com teores adequados a alto de P no solo, tendem a não resposta à adubação (Valderrama et al., 2009; Valderrama et al., 2011; Santini et al., 2014).
Nas variáveis de fixação de $\mathrm{P}$, houve efeito para as fontes, somente para a variável de P-exp (Tabela 3). Este resultado é explicado pelo fato do SFTEA possuir liberação de P melhor distribuído, mantendo-se maior tempo disponibilizando P (Guareschi et al., 2011), em comparação ao SFTC, devido suas melhores características (Reis Junior \& Silva, 2012). Porém, notase, que ao término da condução da cultura, o P no solo residual, não diferiu entre as fontes avaliadas (Tabela 2). Destarte, pode-se inferir que o SFTEA permanecer apenas em curto período disponibilizando maiores teores de $\mathrm{P}$, e, se o mesmo não for absorvido nesse período, é igualmente fixado aos coloides do solo, ou seja, o revestimento do fertilizante apenas prolongaria o tempo para a transformação do P-lábel para o P-não lábel.

As variáveis de dinâmica de $\mathrm{P}$ (P-res; P-exp; e Pfix), nas doses de $\mathrm{P}$ avaliadas, diferente das demais variáveis analisadas (componentes produtivos e produtividade de grãos), optou-se por realizar análises de regressão polinomial de até terceiro grau (Tabela 2), está opção se fez necessária, em razão da notória ineficiência de explicação por meio de equações de menores graus.

Verificou-se que o P-exp pouco influenciou o balanço geral de P no solo, todavia, observa-se que o aumento na exportação de $\mathrm{P}$ não é estabelecida já nas doses iniciais de $\mathrm{P}\left(\right.$ doses $<33 \mathrm{~kg} \mathrm{ha}^{-1}$ por $\mathrm{kg}$ de $\mathrm{P}_{2} \mathrm{O}_{5}$ ), necessitando maiores reposições de $\mathrm{P}$ ao solo, para que a planta absorva e redistribua o $\mathrm{P}$ nos tecidos vegetais. Já o P-res e P-fix foram bastantes influenciados pela adubação e possuem dependência entre eles.

O P-res, até a dose, aproximada, de $75 \mathrm{~kg} \mathrm{ha}^{-1} \mathrm{de}$ $\mathrm{P}_{2} \mathrm{O}_{5}$ foi estático, ou seja, não ocorreu aumento nos teores de P disponíveis no solo, sendo explicado pelo o P-fix, que até a mesma dose, sua concentração aumentou, ou seja, maiores quantidades de $\mathrm{P}$ foram fixados (P não-lábil), não sendo passiveis de absorção. 
Em decorrente à dose de $75 \mathrm{~kg} \mathrm{ha}^{-1}$ de $\mathrm{P}_{2} \mathrm{O}_{5}$, o P-fix reduz sua atividade, ocorrendo o aumento nos teores de P-res. Com o P-fix retornando sua atividade a partir da dose de $155 \mathrm{~kg} \mathrm{ha}^{-1}$ de $\mathrm{P}_{2} \mathrm{O}_{5}$, resultando em valores menos expressivos de P-res, promovendo com que, na dose de $193 \mathrm{~kg} \mathrm{ha}^{-1}$ de $\mathrm{P}_{2} \mathrm{O}_{5}$, seja o ponto de máxima (estimada) de P-res.

Partindo dos dados obtidos nas análises da dinâmica do $\mathrm{P}$ (Figura 1), torna-se evidente que o uso de doses inferiores a $75 \mathrm{~kg} \mathrm{ha}^{-1}$ de $\mathrm{P}_{2} \mathrm{O}_{5}$ resulta na degradação do solo, pela não reposição do P inicial, para as condições do presente trabalho. Mas, deve salientar, que fixação de $\mathrm{P}$ pode variar de acordo com a textura do solo (Machado \& Souza, 2012) e até os minerais constituintes de suas frações (Vilar et al., 2010; Barbieri et al., 2013).

De modo geral, para as fontes avaliadas, o SFTEA não proveu melhorias para os componentes de agronômicos e produtividade (Tabela 1), bem como, não resultou em menor fixação de $\mathrm{P}$ (Tabela 2).

Em estudos com o uso de fontes fosfatadas de eficiência aumentada, encontra-se divergência na real eficácia dessa tecnologia. Quando significativo o efeito, os fertilizantes de eficiência aumentada são superiores aos fertilizantes convencionais, como observado por Figueiredo et al., 2012; Santini et al., 2013; e Souza et al., 2014, mas, também, há trabalhos com resultados semelhantes do presente estudo, sem melhorias pelo uso de fertilizantes de maiores eficiências, como concluído por Vaderrama et al., 2009; Vaderrama et al., 2011; e Gazola et al., 2013.

Não se sabe ao certo os fatores que influenciam na responsividade dos fertilizantes de maior eficiência, contudo, quando analisado os trabalhos supracitados, nota-se que nos trabalhos com efeito significativos, os teores de $\mathrm{P}$ do solo variou de 0,2 a 7,83 $\mathrm{mg} \mathrm{dm}^{-3} \mathrm{e}$ utilizou-se o método de extração de P de Mehlich I, já os trabalhos sem efeitos, os teores de $\mathrm{P}$ variaram de 18 a $31 \mathrm{mg} \mathrm{dm}^{-3}$ e utilizou-se o método de extração de $\mathrm{P}$ de resina trocadora de íons. Salientando, que no presente trabalho, o solo utilizado possuía elevado teor de $\mathrm{P}$, todavia, utilizou-se para avaliação do teor $\mathrm{P}$ do solo, o método de extração de P de Mehlich I.

Não se pode afirmar, categoricamente, que o teor de P no solo e/ou o método de extração são os responsáveis pelos efeitos verificados no uso de fertilizantes de eficiência aumentada, porém, podem estar associados a tal feito. Assim, os fertilizantes de eficiência aumentada seriam mais responsivos em solos com menores teores de P.

\section{CONCLUSÃO}

O Superfosfato triplo convencional é a melhor opção de aplicação, comparativamente ao superfosfato triplo de eficiência aumentada, devido a não diferença entre as fontes e ao fato do fertilizante convencional possuir melhor relação custo-benefício.

A cultivar em estudo não possui melhoria na produtividade de grãos com a adubação fosfatada, em doses elevadas de aplicação, em solo com teor alto de P.

$\mathrm{O}$ uso de $75 \mathrm{~kg} \mathrm{ha}^{-1}$ de $\mathrm{P}_{2} \mathrm{O}_{5}$ foi suficiente para suprir a necessidade da cultura e manter o nível inicial de $\mathrm{P}$ do solo. Doses inferiores a $75 \mathrm{~kg} \mathrm{ha}^{-1} \mathrm{de}_{2} \mathrm{O}_{5}$ não acarretarão na reposição nutricional de $\mathrm{P}$, o que, por consequência, poderá levar a degradação química desse meio, ao passo dos anos de cultivos.

\section{REFERÊNCIAS}

Alcântara Neto F, Gravina GA, Souza NOS, Bezerra AAC. Adubação fosfatada na cultura da soja na microrregião do Alto Médio Gurguéia. Revista Ciência Agronômica. 2010;41(2):266- 271.

Araújo FF. Disponibilização de fósforo, correção do solo, teores foliares e rendimento de milho após a incorporação de fosfatos e lodo de curtume 
natural e compostado. Acta Scientiarum. 2011; $33(2): 355-360$

Barbieri D M, Marques Jr J, Pereira GT, Scala Jr NL, Siqueira DS, Panosso AR. Comportamento dos Óxidos de ferro da Fração argila e fazer adsorvido Fósforo, EM Diferentes Sistemas de Colheita de cana-de-açúcar. Revista Brasileira de Ciência do Solo. 2013;37(6):1557-1568.

Blaylock A. O futuro dos fertilizantes nitrogenados de liberação controlada. Informações agronômicas. 2007;120(1):08-10.

Buratto JS. Teores de minerais e proteina em grãos de feijão e estimativa de parâmetros genéticos. 147f. Tese (Doutorado) - Lavras : Universidade Federal de Lavras; 2012.

Caixeta DS. Seleção precoce e indireta para eficiência no uso de nitrogênio em milho. 58f. Dissertação (Mestrado em Fitotecnia) - Viçosa: Universidade Federal de Viçosa; 2012.

Carvalho VGB, Nascimento CWA, Biondi CM. Potencial de fertilizantes e corretivos no aporte de micronutrientes ao solo. Revista Brasileira de Ciência do Solo. 2012; 36:931-938.

Duncan RR, Baligar VC. Genetics, breeding, and physiological mechanisms of nutrient uptake and use efficiency: An overview. In: BALIGAR VC, DUNCAN RR (eds). Crops as enhancers of nutrient use. Academic Press, Nova York, Estados unidos. 588 p.; 1990

Empresa Brasileira de Pesquisa Agropecuária (EMBRAPA). Tecnologias de produção de soja - região central do Brasil 2012 e 2013. EMBRAPA, Londrina, Brasil. 261 p.; 2011.

Ferreira DF.Sisvar: a computer statistical analysis system. Ciência e Agrotecnologia. 2011; 35(6):10391042.

Figueiredo CC, Barbosa DV, Oliveira AS, Fagioli M, Sato JH. Adubo fosfatado revestido com polímero e calagem na produção e parâmetros morfológicos de milho. Revista Ciência Agronômica. 2012; 43(3):446-452.

Gazola RN, Buzetti S, Dinalli RP, Teixeira Filho MCM, Celestrino TS. Efeito residual da aplicação de fosfato monoamônio revestido por diferentes polímeros na cultura de milho. Revista Ceres. 2013; 60(6):876-884.

Guareschi RF, Gazolla PR, Perin A, Santini JMK. Adubação antecipada da cultura da soja com superfosfato triplo e cloreto de potássio resvetidos por polímeros. Ciência e Agrotecnologia. 2011; 35(4):643-648.

Korndörfer GH, Sousa RTX. Recuperação do fósforo aplicado no solo de fontes solúveis e insolúveis em água. Enciclopédia biosfera, Centro Cientifico Conhecer. 2010; 6(11):9-15.

Lindsay WL. Inorganic phase equilibria of micronutrients in soils. In: Mortvedt JJ, Giordano PM, Lindsay WL (eds.) Micronutrients in agriculture. Soil Science Society of America, Madison, USA. 760 p.; 1972.

Machado VJ, Souza CHE. Disponibilidade de fósforo em solos com diferentes texturas após aplicação de doses crescentes de fosfato monoamônico de liberação lenta. Bioscience Journal. 2012; 28:1-7.

Malavolta E, Vitti GC, Oliveira AS. Fundações. In: Malavolta E, Vitti GC, Oliveira SA (Ed.). Avaliação do estado nutricional das plantas: princípios e aplicações. 2 ed.: Associação Brasileira para a Pesquisa da Potassa e do Fosfato, Piracicaba, Brasil. 319 p.; 1997.

Marschner H. Nitrogen fixation. In: Marschner H. (ed.) Mineral nutrition of higher plants. 2 ed. Academic Press, Londres, Inglaterra, 201-228p.; 1995.

Oliveira LB, Tiecher T, Quadros FLF, Santos DR. Fósforo microbiano em solos sob pastagem natural submetida à queima e pastejo. Revista Brasileira de Ciência do Solo. 2011; 35:1509-1515.

Van Raij B, Andrade JC, Cantarella H, Quaggio JÁ. Análise química para avaliação da fertilidade de solos tropicais. Instituto Agronômico de Campinas, Campinas, Brasil. 285 p.; 2001.

Reis Jr RA, Silva DRG. Avaliação das características físicas e físico-químicas de fertilizantes nitrogenados e fosfatados revestidos por polímeros. Magistra. 2012; 24(2):145-150.

Richart A, LanaMC, Schulz LR, Bertoni JC, Braccini AL. Disponibilidade de fósforo e enxofre para a cultura da soja na presença de fosfato natural reativo, superfosfato triplo e enxofre elementar. 
Revista Brasileira de Ciência do Solo. 2006; 30(4):695705.

Santini JMK, Castro CFS, Perin A, Furquim CS, Furquim LC. Teor nos grãos e qualidade do óleo de soja em resposta a fontes e doses de adubo fosfatado. Cultura Agronômica. 2014; 23(2):101108.

Santini JMK, Perin A, Silveira FO, Lopes Filho LC, Valderram M. The usage of NPK coated by polymers on the cotton crop (GOSSYPIUM HIRSUTUM L.) For biomass production on the aerial part. Global Science and Technology. 2013; 6(3):79-89.

Siqueira JO, Moreira FMS, Grisi BM, Hungria M, Araujo RS. Microrganismos e processos biológicos do solo: perspectiva ambiental. EMBRAPA, Brasília, Brasil. 142 p.; 1994.

Sousa DMG, Lobato E. CERRADO: Correção do solo e adubação, $2^{\mathrm{a}}$ Edição EMBRAPA, Brasília, Brasil 416 p.; 2004.
Souza JR, Ribeiro BN, Raposo TP, Fiorin JE, Castro GSA, Magalhães RS. Eficiência do fósforo revestido com polímeros na cultura da soja Acta Igиағи. 2014; 3(4):1-9

Valadão Júnior DD, Bergamin AC, Venturoso LR, Schlindwein JA, Caron BO, Schmidt D. Adubação fosfatada na cultura da soja em Rondônia Scientia Agraria. 2008; 9(3):369-375

Valderrama M, Buzetti S, Benett CGS, Andreotti M, Alf $\mathrm{O}$, Eustaquio SM. Fontes e doses de nitrogênio e fósforo em feijoeiro no sistema plantio direto Pesquisa Agropecuária Tropical. 2009; 39(3):191-196

Valderrama M, Buzetti S, Benett CGS, Andreotti M, Teixeira Filho MCM. Fontes e doses de NPK em milho irrigado sob plantio direto Pesquisa Agropecuária Tropical. 2011; 41:(2):254-263

Vilar CC, Costa ACS, Hoepers A, Souza Junior IG. Capacidade máxima de adsorção de fósforo relacionada a formas de ferro e alumínio em solos subtropicais Revista Brasileira de Ciência do Solo. 2010; 34:1059-1068 